\title{
Direct Observation of Ion Dynamics in Supercapacitor Electrodes using in situ Diffusion NMR Spectroscopy
}

\author{
Alexander C. Forse, ${ }^{1,2}$ John M. Griffin,, ${ }^{1,3}$ Céline Merlet, ${ }^{1}$ Javier Carretero-Gonzalez, ${ }^{1}$ Abdul-Rahman O. Raji, ${ }^{1,4}$ \\ Nicole M. Trease, ${ }^{1}$ Clare P. Grey ${ }^{1, *}$ \\ ${ }^{1}$ Department of Chemistry, University of Cambridge, Lensfield Road, Cambridge, CB2 1EW, U.K. \\ ${ }^{2}$ Current Address: Department of Chemistry, Department of Chemical and Biomolecular Engineering, and Berkeley Energy and \\ Climate Institute, University of California, Berkeley, USA. \\ ${ }^{3}$ Current Address: Department of Chemistry, Lancaster University, Lancaster, LA1 4YB, U.K. \\ ${ }^{4}$ Cambridge Graphene Centre, University of Cambridge, Cambridge CB3 OFA, U.K. \\ *Corresponding author:.cpg27@cam.ac.uk
}

Ionic transport inside porous carbon electrodes underpins the storage of energy in supercapacitors and the rate at which they can charge and discharge, yet few studies have elucidated the materials properties that influence ion dynamics. Here we use in situ pulsed field gradient NMR spectroscopy to measure ionic diffusion in supercapacitors directly. We find that confinement in the nanoporous electrode structures decreases the effective self-diffusion coefficients of ions by over two orders of magnitude compared to neat electrolyte, and in-pore diffusion is modulated by changes in ion populations at the electrode-electrolyte interface during charging. Electrolyte concentration and carbon pore size distributions also affect in-pore diffusion and the movement of ions in and out of the nanopores. In light of our findings we propose that controlling the charging mechanism may allow the tuning of the energy and power performances of supercapacitors for a range of different applications.

As renewable energy and green technologies such as electric vehicles become prevalent, we must develop new ways to store and release energy on a range of timescales. Rechargeable batteries are ideal for timescales of minutes or hours (electric cars, portable electronic devices, grid storage etc.), while supercapacitors are more promising for second or sub-second timescales and are increasingly being used for transport applications where rapid charging and discharging are required. The superior power handling and cycle lifetime of supercapacitors comes at the expense of energy density, with recent materials-driven research aiming to address this issue by fine-tuning the nanoporous structure of the carbon electrodes, ${ }^{1,2}$ and by using ionic liquid electrolytes that are stable at higher voltages. ${ }^{3,4}$ Both approaches have afforded some increases in energy density, though not without sacrificing power density. The delicate balance between energy and power density must be understood if supercapacitors are to be used in a wide range of applications.

Fundamental studies based on spectroscopic, ${ }^{5-14}$ and theoretical, ${ }^{15-18}$ methods have recently revealed the complex nature of charging in supercapacitors. Prior to charging, the electrode pores contain a large number of electrolyte ions, ${ }^{15,19,20}$ and as a result charge storage is generally more complex than simple counter-ion adsorption (counter-ions are defined as having charge opposite to the electrode in which they are located). ${ }^{5-7,15}$ A range of different charging mechanisms can operate 
depending on the choice of electrode and electrolyte, and the electrode polarisation. ${ }^{21,22}$ For example, charging often proceeds via the exchange of counter-ions and co-ions (co-ions are defined as having charge of the same sign as the electrode in which they are located), while charging by coion desorption alone is also a possibility. We introduced the charging mechanism parameter, $X$, to quantify these different processes, with $X$ taking values of $+1,0$ and -1 , for the extreme cases of charging by counter-ion adsorption, counter-ion - co-ion exchange, and co-ion desorption, respectively, while intermediate $X$ values indicate contributions from more than one mechanism. ${ }^{21}$

An understanding and control of the charge storage mechanism ( $X$ value) may hold the key to optimising the energy and power performance of supercapacitors for different applications. However, a crucial missing part of our understanding is how the electrolyte ions diffuse and migrate in supercapacitor electrodes. Theoretical studies based on molecular dynamics (MD) and meanfield theories have shown that ion-ion interactions, ion-carbon interactions and the electrode pore size all influence the rates of ionic diffusion in supercapacitor electrodes, ${ }^{16,18,23,24}$ though there is not yet a clear consensus on which factors are most important, nor the order of magnitude by which diffusion is influenced by confinement and by charging. Experimental methods to directly probe inpore motion in an ion-selective and electrode-selective way have until now been lacking.

Here we show how an in situ pulsed field gradient (PFG) NMR approach can be used to measure ionic diffusion in the nanopores of supercapacitor electrodes. Confinement results in reductions in self-diffusion coefficients by over two orders of magnitude compared to neat electrolyte solutions, while in situ measurements show that changes of in-pore ion populations during charging modulate in-pore ionic diffusion. We also show that the electrolyte concentration and nanopore size have significant effects on in-pore diffusion, and the exchange of ions between bulk and in-pore sites. Our findings offer detailed insights into diffusion and exchange processes in porous electrodes and bring new opportunities for understanding and controlling the charging dynamics of supercapacitors. 


\section{Results}

a)

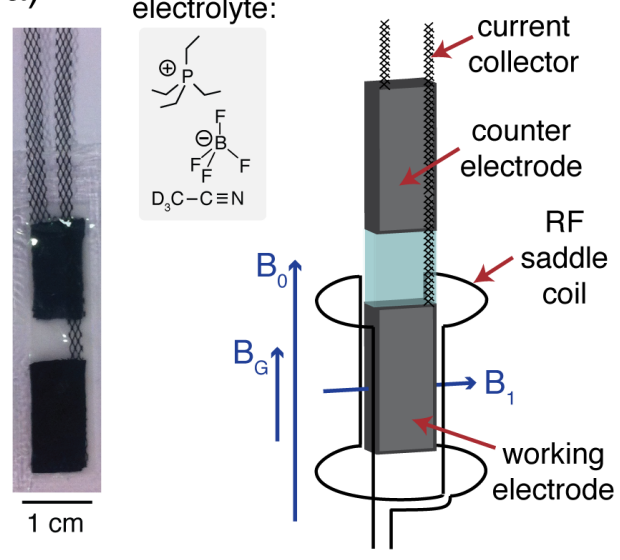

b)

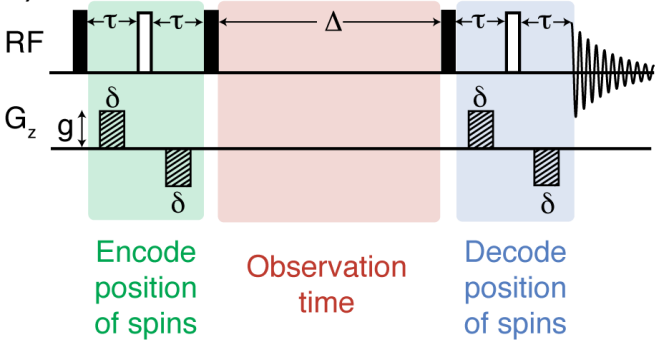

c)

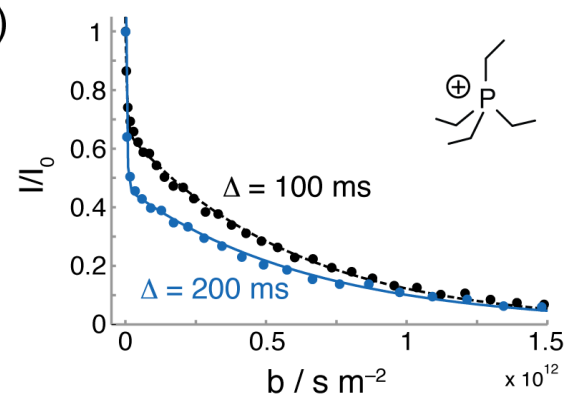

Figure 1: Introducing Pulsed Field Gradient NMR of Supercapacitors. a)

Photograph of pouch cell, electrolyte, and schematic of in situ PFG set-up showing the orientation of the magnetic field, $B_{0}$, magnetic field gradients, $B_{\mathrm{G}}$, and the radiofrequency (RF) field, $B_{1}$ (see SI for discussion of field orientations). b) 13-interval sequence used for PFG NMR experiments, radiofrequency (RF) and magnetic field gradient $\left(\mathrm{G}_{\mathrm{z}}\right)$ pulses are shown. c) Example decays of normalised inpore cation signal intensity against $b$ for a YP50F supercapacitor (cell voltage $=0 \mathrm{~V}$ ) with 1.5 $\mathrm{M} \mathrm{PEt}_{4} \mathrm{BF}_{4}$ in deuterated acetonitrile electrolyte. The parameter $b$ is given by: $b=$ $g^{2}(\gamma 2 \delta)^{2} t_{\text {eff }}=g^{2}(\gamma 2 \delta)^{2}(\Delta-1 / 6 \delta-1 / 2 \tau)$, with $\gamma$ the gyromagnetic ratio of the nucleus under study, $\delta$ the duration of an individual magnetic field gradient pulse, $\tau$ the separation of the 90 and $180^{\circ} \mathrm{RF}$ pulses. The time constants of the biexponential fits give selfdiffusion coefficients, $D$.
Two sets of pouch cell supercapacitors ${ }^{25}$ constructed with electrodes fabricated from two different commercial activated carbons (YP50F and YP80F) with different porosities and $1.5 \mathrm{M}$ tetraethylphosphonium tetrafluoroborate $\left(\mathrm{PEt}_{4} \mathrm{BF}_{4}\right)$ electrolyte (in deuterated acetonitrile, $\mathrm{D}_{3} \mathrm{CCN}$ ) were studied by PFG NMR. In the experiments the cell working-electrode is detected (Figure 1a). Uniquely, NMR allows the separate signals from ions that are inside the carbon nanopores, "inpore", and ions that are outside the nanopores in electrolyte reservoirs, "ex-pore", to be resolved. ${ }^{19,26,27}$ Cations and anions were studied using ${ }^{1} \mathrm{H}$ and ${ }^{19} \mathrm{~F}$ NMR, respectively (see SI for example spectra).

In PFG NMR experiments magnetic field gradient pulses are used to encode and decode the positions of the nuclear spins, with ionic diffusion probed over a given observation time, $\Delta$ (Figure 1b). For each diffusion measurement, a series of NMR spectra are acquired with different magnetic field gradient strengths, $g$, and the normalised signal intensity $\left(I / I_{0}\right)$ is plotted against a parameter, $b$, that is proportional to $g^{2}$ (see Figure 1 caption) ${ }^{28,29}$ Self-diffusion coefficients, $D$, are then obtained by exponential fits of the form $\left(I / I_{0}\right)=\exp (-$ $\mathrm{D} b$ ). For in-pore ions biexponential decays were observed (Figure 1c), indicating the presence of two sets of in-pore ions with different $D$ values. The initial steep decay (large $D$ ) arises from ions that undergo in-pore $\leftrightarrow$ ex-pore exchange processes during the observation time (see SI). 2D exchange NMR measurements confirmed the presence of such exchange (see later). The more gradually decaying component in Figure 1c (small $D$ ), on the other hand, arises from ions that remain in the nanopores during the observation time, with a self- 
diffusion coefficient, $D_{\text {in-pore, }}$ and mole fraction, $A_{\text {in-pore }}$. By performing PFG measurements with different $\Delta$ times, in-pore diffusion and in-pore $\leftrightarrow$ ex-pore exchange processes can be probed on different timescales (and therefore different lengthscales).

For the YP50F supercapacitor at $0 \mathrm{~V}, D_{\text {in-pore }}$ is reduced by over two orders of magnitude compared to neat electrolyte, for all observation times, $\Delta$ (Figure $2 \mathrm{a}$ ). For example, for a relatively short observation time of $\Delta=20 \mathrm{~ms}, D_{\text {in-pore }}$ is reduced by a factor of 300 for cations and 180 for anions. These marked reductions could arise from a number of factors: (i) the local reduction in in-pore diffusion due to collisions with the rigid pore walls, ${ }^{30,31}$ (ii) tortuosity arising from the disordered arrangement of pores ${ }^{32-34}$ in the carbon particles such that ions diffuse in an indirect way, and (iii) the structure and composition of the electrolyte in the pores may differ from neat electrolyte, our previous studies having shown that ions are partially desolvated in the pores. ${ }^{6}$ The difference in diffusion coefficients between anions and cations is amplified upon confinement in the pores (Figure 2a), highlighting the important role of ion size, with the larger cations presumably taking more indirect pathways through the pore network (as the smallest pores are inaccessible). ${ }^{30}$ For a second activated carbon, $Y P 80 F, D_{\text {in-pore }}$ for cations is $\sim 4$ to 5 times larger compared to YP50F depending on the observation time, showing the crucial influence of carbon structure on in-pore diffusion. This increase in $D_{\text {in-pore }}$ is ascribed to the additional porosity with pore widths between 1 and $3 \mathrm{~nm}$ in YP80F (Figure 2b), with these large pores acting as "highways" connecting the smaller pores. Indeed, a previous study showed that the diffusion coefficients of small molecules increased with the pore width of activated carbons. ${ }^{30}$ 


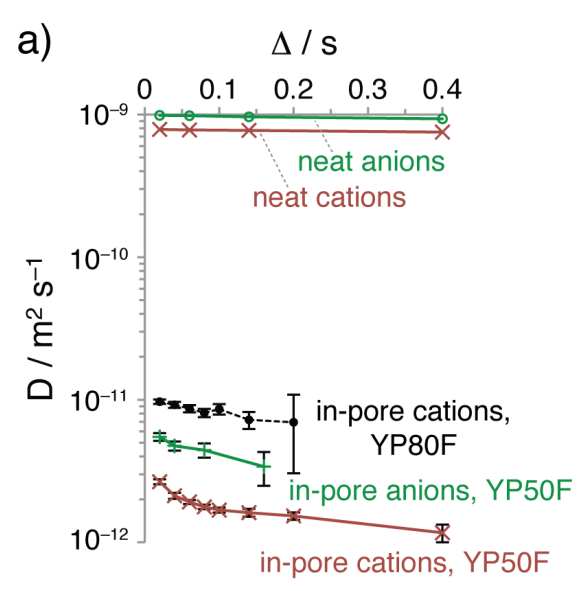

d)

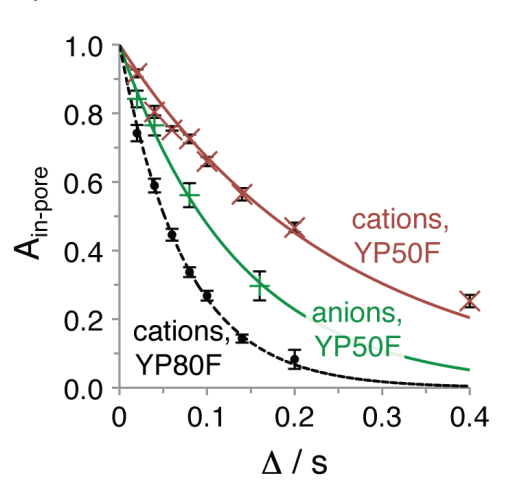

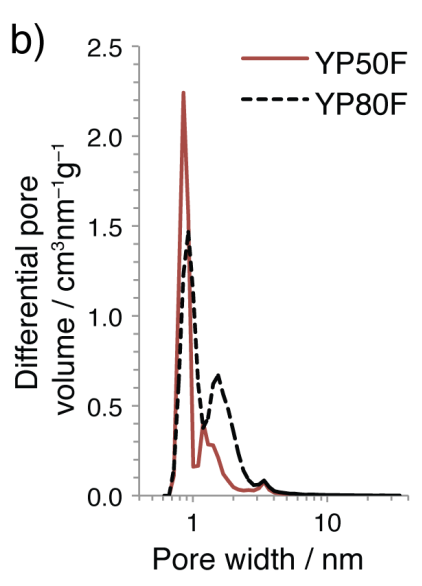

c)

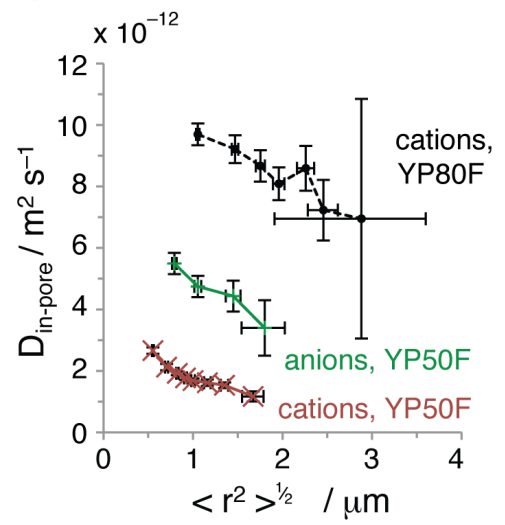

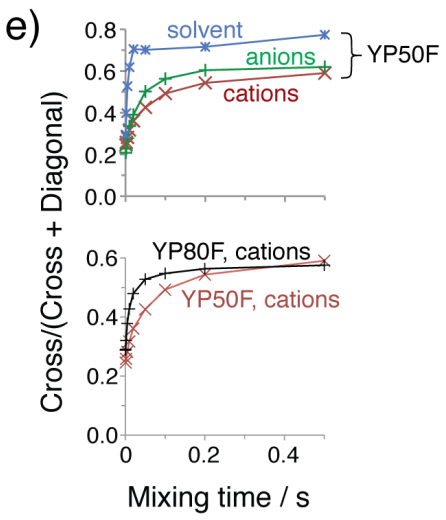

Figure 2: Diffusion measurements on supercapacitors at $0 \mathrm{~V}$. a) Diffusion measurements as a function of $\Delta$, for YP50F and YP80F supercapacitors, with $1.5 \mathrm{M} \mathrm{PEt}_{4} \mathrm{BF}_{4}$ in deuterated acetonitrile electrolyte. Measurements on neat electrolyte are also shown. b) Pore size distributions of the two carbons. c) $D_{\text {in-pore }}$ as a function of root mean square displacement. d) $A_{\text {in-pore }}$ as a function of $\Delta$. Exponential fits allow the mean lifetime of an in-pore ion, $\tau_{\text {in-pore, }}$, to be determined. e) Intensity ratios from $2 \mathrm{D}$ exchange NMR spectroscopy experiments with different mixing times, ${ }^{1} \mathrm{H},{ }^{19} \mathrm{~F}$ and ${ }^{13} \mathrm{C}$ NMR were used to probe cations, anions and isotopically labelled solvent, respectively (see SI). Error bars in a), c) and d) represent $95 \%$ confidence bounds (see Methods).

Our experiments measure in-pore diffusion on relatively long timescales $(20-400 \mathrm{~ms})$, with corresponding lengthscales of several hundreds of nanometres to micrometres (Figure 2c). Root

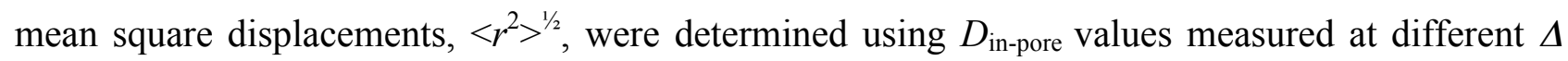
times; $\left\langle r^{2}\right\rangle^{1 / 2}=\left(6 D_{\text {in-pore }} t_{\text {eff }}\right)^{1 / 2}$, with $t_{\text {eff }}=\Delta-1 / 6 \delta-1 / 2 \tau \approx \Delta . D_{\text {in-pore }}$ decreases as larger root mean square displacements, $\left\langle r^{2}\right\rangle^{1 / 2}$, are probed (Figure $2 \mathrm{c}$ ), indicative of restricted diffusion, ${ }^{28}$ with ions becoming more likely to encounter the smallest pores and bottlenecks for longer observation times (longer $\left\langle r^{2}\right\rangle^{1 / 2}$ ). This shows that the structure of the carbon particles is heterogeneous to ionic motion on lengthscales as large as 2 microns, i.e. on the order of the carbon particle sizes (see SI). This is consistent with a recent NMR study of solvents in a nanoporous carbon, ${ }^{35}$ and helps to explain why we observe greater reductions of effective diffusion upon confinement than in MD simulations where much smaller observation times were studied. ${ }^{23}$

PFG NMR experiments provide additional dynamic information about the exchange (or swapping) of ions between in- and ex-pore environments (Figure 2d). $A_{\text {in-pore }}$ gives the mole fraction of in-pore ions that have remained in the nanopores during the observation time, $\Delta$. For YP50F, the anions 
undergo significantly more rapid exchange than the cations, with mean in-pore lifetimes, $\tau_{\text {in-pore }}{ }^{29,36}$ of $135 \pm 14$ and $252 \pm 26 \mathrm{~ms}$, respectively. This suggests that in-pore diffusion limits in-pore $\leftrightarrow$ expore exchange, with the faster anion diffusion resulting in more rapid exchange. Indeed, the larger in-pore cation diffusion coefficient in YP80F electrodes results in even more rapid exchange, with $\tau_{\text {in-pore }}=74 \pm 3$ ms. A measure of the mean carbon particle radius is obtained by $\left(6 D_{\text {in-pore }} \tau_{\text {in-pore }}\right)^{1 / 2}$, giving $1.5 \mu \mathrm{m}$ and $1.7 \mu \mathrm{m}$, for YP50F (probed by the cations and anions, respectively) and $1.9 \mu \mathrm{m}$ for YP80F (cations), with the values consistent with typical carbon particle dimensions (see SI). The build up of cross peak intensity in 2D exchange NMR experiments offer a second probe of inpore $\leftrightarrow$ ex-pore exchange (Figure 2e), ${ }^{7,9}$ and corroborate the results from PFG NMR. The exchange of the smaller, neutral, acetonitrile solvent molecules (as probed by ${ }^{13} \mathrm{C}$ NMR experiments on isotopically enriched acetonitrile) is more rapid than for the ions, indicating that their in-pore diffusion coefficients are larger. This is unsurprising since diffusion measurements on neat electrolyte showed that the solvent diffuses 2.0 times faster than the anions, and 2.5 times faster than the cations. MD simulations have also shown that (de)solvation of in-pore ions with acetonitrile takes place as quickly as $\sim 1 \mathrm{ps},{ }^{23}$ and a recent study of dimethyl carbonate showed a relatively modest reduction of effective diffusion (by a factor of $\sim 0.2$ ) upon confinement in a nanoporous carbon. ${ }^{35}$ Overall, the slower diffusing ions move through a highly dynamic solvent medium in the nanopores.

In situ pulsed field gradient NMR measurements allow the direct measurement of in-pore diffusion in supercapacitors during charging (Figure 3a,b). Measurements were made under equilibrium conditions at different fixed cell voltages. For negative polarisations, marked decreases of $D_{\text {in-pore }}$ are observed for anions and cations, while for positive polarisations only minor changes are observed, with only slight increases in $D_{\text {in-pore }}$ for anions. For the $1.5 \mathrm{M}$ electrolyte, comparing between 0 and $-1.5 \mathrm{~V}, D_{\text {in-pore }}$ decreases by a factor of 3.6 for the anions (averaged across observation times, see SI), while the cations show a similar, but slightly smaller, reduction factor of 2.9.

The variations of $D_{\text {in-pore }}$ with voltage may be understood by examining the changes of in-pore ionic populations at different voltages (Figure 3c). We previously showed that charging in the positive electrode takes place by swapping of anions and cations (counter-ion - co-ion exchange mechanism, $X=0)$, while charging in the negative electrode occurs by counter-ion adsorption $(X=$ +1 , see Figure $3 \mathrm{c}$ inset). ${ }^{5}$ As a result, for positive polarisations the total in-pore ion population shows only minor changes with voltage, while for negative polarisation a marked increase is observed (Figure 3c). This increase of in-pore ion population correlates with the reduction of $D_{\text {in-pore }}$ for both ions: i.e., it appears that increasing ion-ion interactions lead to a reduction of $D_{\text {in-pore with }}$ 
voltage for negative polarisations. In principle ion-carbon interactions may also influence $D_{\text {in-pore, }}{ }^{23}$

a)
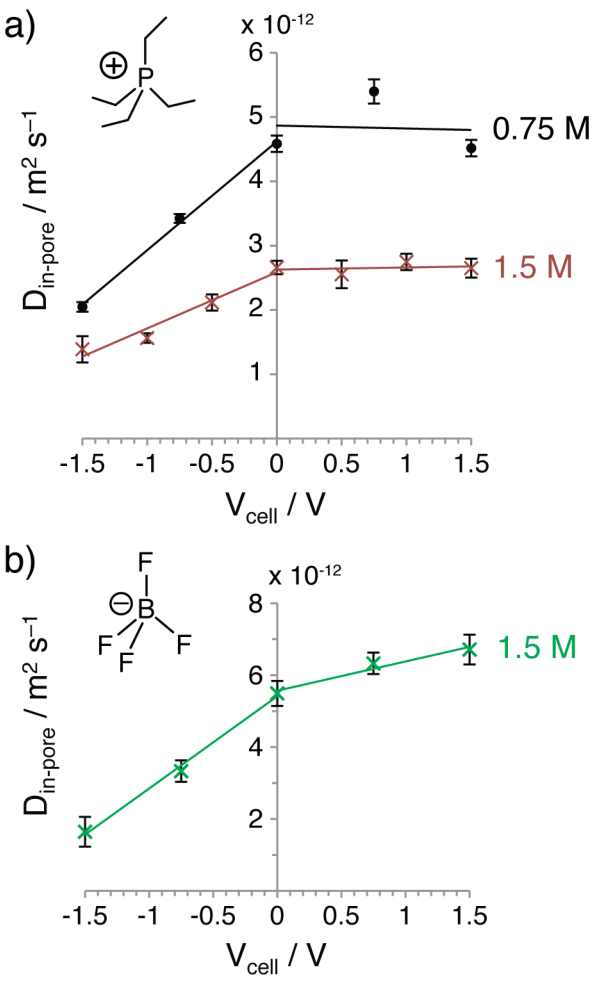

c)

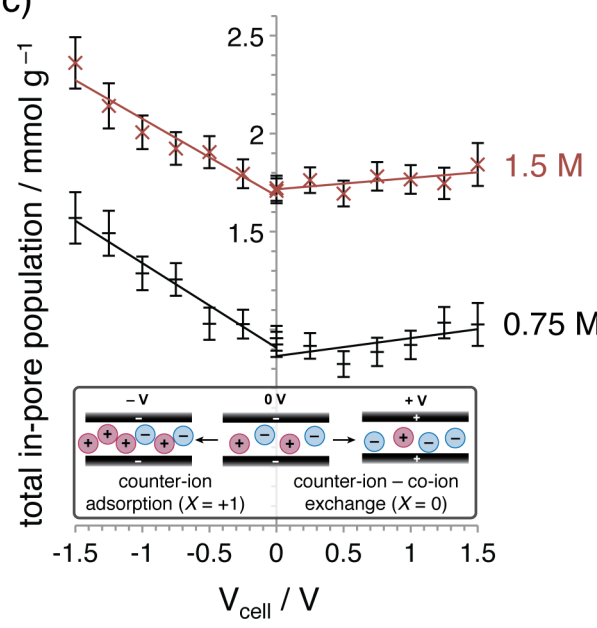

d) $\times 10^{-12}$

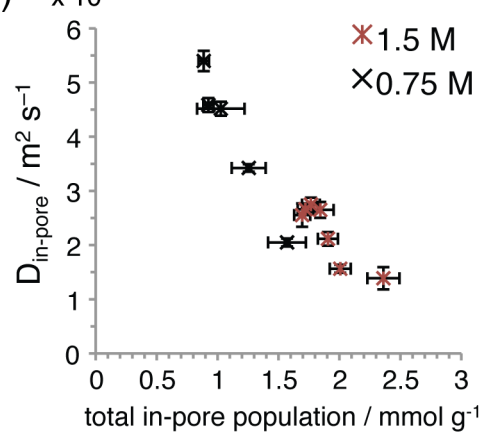

though these appear to be a minor factor, since the anions and cations show such similar variations of $D_{\text {in-pore }}$ with voltage.

To explore the role of ion-ion interactions further, a lower concentration electrolyte $(0.75 \mathrm{M})$ was studied (Figure 3a, c). At $0 \mathrm{~V}$, in-pore cation diffusion is faster by a factor of $\sim 1.7$ for the $0.75 \mathrm{M}$ electrolyte (compared to $1.5 \mathrm{M}$ ), and inpore $\leftrightarrow$ ex-pore exchange is also more rapid, with $\tau_{\text {in-pore }}=$ $160 \pm 7 \mathrm{~ms}$, compared to $252 \pm 26 \mathrm{~ms}$ for $1.5 \mathrm{M}$ (see SI). Measurements at different voltages show that in-pore cation diffusion is consistently faster for the $0.75 \mathrm{M}$ electrolyte. This can be rationalised by the smaller total in-pore ion populations for the $0.75 \mathrm{M}$ electrolyte (Figure $3 \mathrm{c}$ ), and the resulting reduction in ion-ion interactions. The changes of $D_{\text {in-pore }}$ with voltage are very similar for the two concentrations (Figure 3a), which is due to the similar changes of in-pore population with voltage (the charging mechanisms are very similar for the two concentrations). ${ }^{5} \mathrm{~A}$ global plot for the cations in Figure $3 \mathrm{~d}$ further highlights the inverse correlation between $D_{\text {in-pore }}$ and the total in-pore ion population. It should be noted that the variations of in-pore anion:cation ratios with voltage are different for the two concentrations, so we do not expect the 1.5 and $0.75 \mathrm{M}$ datasets to overlap exactly in Figure $3 \mathrm{~d}$. We finally note that the gravimetric electrode capacitances (see SI) are 107 and $106 \mathrm{~F} \mathrm{~g}^{-1}$ for the 1.5 and $0.75 \mathrm{M}$ cells, respectively. These are identical within error such that the variation of charge with voltage is very similar for the two cells.

Figure 3: In situ measurements of ionic diffusion in supercapacitors. a) Cation and b) anion diffusion in YP50F $\mathrm{PEt}_{4} \mathrm{BF}_{4}(1.5 \mathrm{M})$ in acetonitrile supercapacitors, at different cell voltages. In a) data for a supercapacitor with $0.75 \mathrm{M}$ electrolyte are also shown c) Total in-pore ion populations at different potentials, determined from data in Ref. [5]. Inset shows schematic of charge storage mechanism for this system. d) Inverse correlation between $D_{\text {in-pore }}$ (cations) and total in-pore ion population. All data are for $\Delta=20 \mathrm{~ms}$ (data with $\Delta=40 \mathrm{~ms}$ show very similar trends, see SI). Error bars in a), b) and d) represent 95\% confidence bounds (see Methods). Error bars in c) represent the range of values obtained from four independent fits of each dataset (see Ref. 5). 


\section{Discussion}

Our in situ results strongly suggest that changes of in-pore ion populations, and the resulting changes in the number of ion-ion interactions, dominate the variations of in-pore ionic diffusion observed during charging. The ion-ion interactions have at least two contributions: (i) steric effects, with the increased number of ions sterically hindering diffusion, (ii) electronic effects, whereby associations between oppositely charged ions can reduce ionic self-diffusion. ${ }^{37-39}$ The latter effect will vary in a complex way as the proportions of anions and cations vary during supercapacitor charging, and may explain the small increases in $D_{\text {in-pore }}$ for the anions, observed for positive polarisations. The apparent dominance of ion-ion interactions on the voltage dependence of $D_{\text {in-pore }}$ is consistent with a recent MD study of an ionic liquid inside slit-pore electrodes. ${ }^{18}$ A further MD study on disordered electrode structures revealed changes of $D_{\text {in-pore }}$ with voltage on the same order of magnitude as those observed here (though the charging mechanism, and thus in-pore populations differed for their system). ${ }^{23}$ The dependence of $D_{\text {in-pore }}$ on the in-pore ion population has important consequences for supercapacitor charging rates. Depending on the mechanism that operates (ion exchange, counter-ion adsorption, co-ion desorption etc.) the charging dynamics will vary. This offers new opportunities to improve charging dynamics in supercapacitors if the charging mechanism ( $X$ value) can be controlled. If $X>0$, then the in-pore ion population will increase with voltage and $D_{\text {in-pore }}$ is expected to decrease. On the other hand, if $X<0$ (net ion desorption), $D_{\text {in-pore }}$ would be expected to decrease with voltage. For the case of initially filled pores, charging purely by co-ion desorption $(\mathrm{X}=-1)$ may offer the fastest charging rates at high voltages, though this mechanism has not yet been observed. ${ }^{21}$ Kondrat et al. have suggested that ideally the carbon pores would be initially empty of ions (ionophobic pores) to facilitate fast charging, ${ }^{16,24,40}$ though in practice ionophobic pores have not yet been realised experimentally. Ionic liquids with activated carbons represent the opposite extreme as the pores are densely packed with ions in the absence of an applied potential, ${ }^{6,15}$ and our results help to explain their poor power performances.

Our measurements on carbons with different pore sizes help to explain previous electrochemical studies of charging dynamics. ${ }^{1,41-43}$ For carbons with smaller average pore sizes and fewer mesopores $(<2 \mathrm{~nm})$, the capacitance decreased more rapidly as the current density was increased. We postulate that this is because slower in-pore transport in the smaller pores results in nonequilibrium charge storage mechanisms when practical current densities are used, whereby the optimum (lowest voltage) configurations of ions cannot be attained. As a result, the voltage shows a larger variation with charge, and the capacitance decreases. Our findings also help to explain the excellent high-current performances of hierarchical meso/micropore structures $^{41}$ and zeolitetemplated carbons with pore connectivity in three dimensions. ${ }^{44}$ 


\section{Conclusion}

In conclusion, our in situ PFG NMR experiments reveal new insights into ionic diffusion and exchange processes in supercapacitors. Confinement of the ions inside YP50F nanoporous electrodes leads to a large (more than two orders of magnitude) reduction in their effective diffusion coefficients. The important role of ionic size has also been demonstrated, with the larger cations showing the largest reductions of diffusion upon confinement. Our measurements also allow the mean lifetime of in-pore ions to be determined $\left(\tau_{\text {in-pore }}\right)$, and indicate that in-pore diffusion limits the in-pore $\leftrightarrow$ ex-pore exchange process. We have also shown that the structure of the carbon particles is heterogeneous to ionic motion on lengthscales as large as microns.

In situ diffusion measurements at different electrolyte concentrations have shown that ion-ion interactions dominate the observed variations of $D_{\text {in-pore }}$ with voltage, with the different charging mechanisms ( $X$ values) in the two electrodes resulting in drastically different dynamic behaviour. Charging by counter ion adsorption $(X=+1)$ results in decreases of $D_{\text {in-pore }}$ for negative polarisations, while charging by counter-ion - co-ion exchange $(X=0)$ results in much smaller changes of $D_{\text {in-pore }}$ for positive polarisations. Our findings demonstrate the important interplay between charging mechanisms and dynamics, and should offer new opportunities to control the power performance of supercapacitors. One simple way to improve charging rates may be to use lower electrolyte concentrations as this results in faster in-pore diffusion. However, this must be balanced with the availability of electrolyte ions for charging processes, and efforts must also be made to probe any electrolyte concentration gradients ${ }^{45}$ formed during charging. Achieving truly ionophobic pores, or alternatively charging by co-ion desorption, may enhance charging rates. Furthermore, we have shown how the nanoporous structure of the electrode has a significant effect on in-pore ion diffusion and exchange, with the incorporation of a relatively small amount of additional pore volume in the $1-3 \mathrm{~nm}$ range resulting in a large increase of $D_{\text {in-pore }}$ and decreases of $\tau_{\text {in-pore}}$ Further PFG NMR measurements on a range of different porous carbons will further our understanding and could enable precise control over power and energy performance.

Our measurements have provided a wealth of information that can bring new opportunities for the design of enhanced supercapacitors. The findings will facilitate modelling of full supercapacitor devices, such that the interplay between structure and ion transport can be understood across the full range of lengthscales. Future measurements will be made to establish how ionic migration in an electric field differs from equilibrium self-diffusion, ${ }^{46-48}$ such that the non-equilibrium states reached at the fastest charging/discharging rates can be probed. Only then will we begin to fully unravel the relationships between materials properties and performance in supercapacitors. 


\section{Methods}

Freestanding carbon film electrodes (95 wt. \% carbon, thickness $\sim 250 \mu \mathrm{m}$ ) were prepared by mixing YP50F (or YP80F) activated carbon (Kuraray Chemical) with polytetrafluoroethylene (Sigma Aldrich, 60 wt. \% dispersion in water), as previously described. ${ }^{19}$ Film electrodes were dried for at least 15 hours at $200{ }^{\circ} \mathrm{C}$ in vacuo, with supercapacitor devices then prepared in an $\mathrm{Ar}$ glovebox $\left(<0.1\right.$ ppm $\mathrm{H}_{2} \mathrm{O}$ and $\left.\mathrm{O}_{2}\right)$ in plastic pouch cells, with a design adapted from our previous work. $^{25}$ These pouch cells were positioned inside either $10 \mathrm{~mm}$ or $5 \mathrm{~mm}$ NMR tubes for PFG NMR experiments. Electrode masses were equal within $0.2 \mathrm{mg}$ for all cells, and were typically $\sim 20 \mathrm{mg}$ for cells in $10 \mathrm{~mm}$ NMR tubes, and $\sim 7 \mathrm{mg}$ for cells in $5 \mathrm{~mm}$ tubes. All devices used either 1.5 or 0.75 M tetraethylphosphonium tetrafluoroborate ( $>97 \%$ purity, Toyko Chemical Industries) in deuterated acetonitrile $\left(\mathrm{D}_{3} \mathrm{CCN}, 99.8\right.$ atom \%, Euroisotop) as the electrolyte, with the exception of carbon NMR experiments where $\mathrm{H}_{3} \mathrm{C}^{13} \mathrm{CN}$ (99 atom \%, Isotech) was used. Carbon pore size distributions were obtained from quenched solid density functional theory analysis (assuming slit-pores) of $\mathrm{N}_{2}$ gas sorption measurements carried at $77 \mathrm{~K} .^{49}$

Diffusion NMR experiments were carried out using a Bruker Avance spectrometer and a Diff-50 probehead at a magnetic field strength of $7.1 \mathrm{~T}\left({ }^{1} \mathrm{H}\right.$ larmor frequency at $\left.300 \mathrm{MHz}\right)$. Saddle coils (10 $\mathrm{mm}$ inner diameter for ${ }^{1} \mathrm{H}, 5 \mathrm{~mm}$ for ${ }^{19} \mathrm{~F}$ and ${ }^{13} \mathrm{C}$ ) were used for radiofrequency excitation and detection, with the working electrode positioned in the detection area, with the $B_{1}$ field and electrode orientated as indicated in the SI. The 13 -interval pulse sequence ${ }^{50}$ with bipolar pulsed field gradients was used for diffusion measurements to minimise the effects of any magnetic field gradients inherent to the sample. The maximum $g$ used was $17 \mathrm{~T} \cdot \mathrm{m}^{-1}$, and the effective gradient pulse duration, $\delta$, (as defined in Figure 1 b) was $0.5 \mathrm{~ms}$. The in situ PFG NMR methodology is similar to our previous in situ NMR studies of supercapacitors, with spectra acquired under equilibrium conditions during the application of constant cell voltages. ${ }^{25}$ For determination of $D$ and $A$ values, in-pore intensities were first determined by integration in Topspin software and the variation of intensity with the parameter $b$ was analysed using a Matlab script and the curve fitting tool. Plotted error bars in the diffusion parameters represent upper and lower bounds at 95\% confidence limits, as determined using the curve-fitting tool. 2D exchange spectroscopy measurements were performed as in our previous work. ${ }^{7}$ Spectra are referenced as; ${ }^{1} \mathrm{H}$ - methyl group of ethanol at $1.2 \mathrm{ppm},{ }^{19} \mathrm{~F}$ - hexafluorobenzene at $-164.9 \mathrm{ppm}$, and ${ }^{13} \mathrm{C}$ - ethylene group of ethanol at $58.0 \mathrm{ppm}$. RF excitation pulses were calibrated on the supercapacitor cells, with powers of between $22-30 \mathrm{kHz}$ used for all nuclei. Recycle delays were set to $\sim 1.2 T_{1}$ to maximise the signal to noise for a given experiment time. Sufficiently long delays of $0.5 \mathrm{~ms}$ were inserted between PFG 
and RF pulses such that eddy currents did not influence diffusion measurements. Experiments on neat electrolytes were performed in $5 \mathrm{~mm}$ NMR tubes.

\section{References}

1. Chmiola, J. et al. Anomalous increase in carbon capacitance at pore sizes less than 1 nanometer. Science 313, 1760-1763 (2006).

2. Raymundo-Piñero, E., Kierzek, K., Machnikowski, J. \& Béguin, F. Relationship between the nanoporous texture of activated carbons and their capacitance properties in different electrolytes. Carbon 44, 2498-2507 (2006).

3. Balducci, A. Electrolytes for high voltage electrochemical double layer capacitors: A perspective article. $J$. Power Sources 326, 534-540 (2016).

4. Zhong, C. et al. A review of electrolyte materials and compositions for electrochemical supercapacitors. Chem. Soc. Rev. 44, 7484-7539 (2015).

5. Griffin, J. M. et al. In Situ NMR and Electrochemical Quartz Crystal Microbalance Reveal the Structure of the Electric Double-Layer in Supercapacitor Electrodes. Nat. Mater. 14, 812-819 (2015).

6. Forse, A. C. et al. NMR study of ion dynamics and charge storage in ionic liquid supercapacitors. J. Am. Chem. Soc. 137, 7231-7242 (2015).

7. Griffin, J. M. et al. Ion Counting in Supercapacitor Electrodes using NMR Spectroscopy. Faraday Disc. 176, 49-68 (2014).

8. Luo, Z.-X. et al. Dehydration of Ions in Voltage-Gated Carbon Nanopores Observed by in Situ NMR. J. Phys. Chem. Lett. 6, 5022-5026 (2015).

9. Deschamps, M. et al. Exploring electrolyte organization in supercapacitor electrodes with solid-state NMR. Nat. Mater. 12, 351-358 (2013).

10. Tsai, W.-Y., Taberna, P.-L. \& Simon, P. Electrochemical Quartz Crystal Microbalance (EQCM) Study of Ion Dynamics in Nanoporous Carbons. J. Am. Chem. Soc. 136, 8722-8728 (2014).

11. Levi, M. D., Salitra, G., Levy, N., Aurbach, D. \& Maier, J. Application of a quartz-crystal microbalance to measure ionic fluxes in microporous carbons for energy storage. Nat. Mater. 8, 872-875 (2009).

12. Prehal, C. et al. Tracking the structural arrangement of ions in carbon supercapacitor nanopores using in-situ small-angle X-ray scattering. Energy Environ. Sci. 8, 1725-1735 (2015).

13. Richey, F. W., Tran, C., Kalra, V. \& Elabd, Y. A. Ionic Liquid Dynamics in Nanoporous Carbon Nanofibers in Supercapacitors Measured with in Operando Infrared Spectroelectrochemistry. J. Phys. Chem. C 118, 2184621855j (2014).

14. Boukhalfa, S.et al. In-Situ Small Angle Neutron Scattering Revealing Ion Sorption in Microporous Carbon Electrical Double Layer Capacitors In-Situ Small Angle Neutron Scattering Revealing Ion Sorption in Microporous Carbon Electrical Double Layer Capacitors. ACS Nano 8, 2495-2503 (2014).

15. Merlet, C. et al. On the molecular origin of supercapacitance in nanoporous carbon electrodes. Nat. Mater. 11, 306-310 (2012).

16. Kondrat, S., Wu, P., Qiao, R.\& Kornyshev, A. A. Accelerating charging dynamics in subnanometre pores. Nat. Mater. 13, 387-393 (2014).

17. Fedorov, M. V \& Kornyshev, A. A. Ionic Liquids at Electrified Interfaces. Chem. Rev. 114, 2978-3036 (2014).

18. He, Y. et al. Importance of Ion Packing on the Dynamics of Ionic Liquids during Micropore Charging. J. Phys. Chem. Lett. 7, 36-42 (2016).

19. Forse, A. C. et al. Nuclear magnetic resonance study of ion adsorption on microporous carbide-derived carbon. Phys. Chem. Chem. Phys. 15, 7722-7730 (2013).

20. Borchardt, L., Oschatz, M., Paasch, S., Kaskel, S. \& Brunner, E. Interaction of electrolyte molecules with carbon materials of well-defined porosity: characterization by solid-state NMR spectroscopy. Phys. Chem. Chem. Phys. 15, 15177-84 (2013).

21. Forse, A. C., Merlet, C. M., Griffin, J. M. \& Grey, C. P. New Perspectives on the Charging Mechanisms of Supercapacitors. J. Am. Chem. Soc. 138, 5731-5744 (2016).

22. Salanne, M. et al. Efficient storage mechanisms for building better supercapacitors. Nat. Energy 1, 16070 (2016).

23. Pean, C. et al. Confinement, Desolvation, And Electrosorption Effects on the Diffusion of Ions in Nanoporous Carbon Electrodes. J. Am. Chem. Soc. 137, 12627-32 (2015).

24. Kondrat, S.\& Kornyshev, A. A. Pressing a spring: what does it take to maximize the energy storage in nanoporous supercapacitors? Nanoscale Horiz. 1, 45-52 (2016).

25. Wang, H. et al. In situ NMR spectroscopy of supercapacitors: insight into the charge storage mechanism. J. Am. Chem. Soc. 135, 18968-80 (2013).

26. Forse, A. C., Griffin, J. M., Presser, V., Gogotsi, Y. \& Grey, C. P. Ring Current Effects: Factors Affecting the NMR Chemical Shift of Molecules Adsorbed on Porous Carbons. J. Phys. Chem. C 118, 7508-7514 (2014). 
27. Merlet, C., Forse, A. C., Griffin, J. M., Frenkel, D. \& Grey, C. P. Lattice simulation method to model diffusion and NMR spectra in porous materials. J. Chem. Phys. 142, 094701 (2015).

28. Callaghan, P. T. Translational Dynamics \& Magnetic Resonance. (2011).

29. Karger, J., Ruthven, D. M. \& Theodorou, D. N. Diffusion in Nanoporous Materials. (2012).

30. Dubinin, M. M., Vartapetian, R. S., Voloshchuk, A. M., Karger, J. \& Pfeifer, H. NMR Study of Translational Mobility of Molecules Adsorbed on Active Carbons. Carbon 26, 515-520 (1988).

31. Kalugin, O. N., Chaban, V. V, Loskutov, V. V \& Prezhdo, O. V. Uniform Diffusion of Acetonitrile inside Carbon Nanotubes Favors Supercapacitor Performance 2008. Nano Lett. 8, 2126-2130 (2008).

32. Oschatz, M. et al. Nanostructure characterization of carbide-derived carbons by morphological analysis of transmission electron microscopy images combined with physisorption and Raman spectroscopy. Carbon 105, 314-322 (2016).

33. Forse, A. C. et al. New Insights into the Structure of Nanoporous Carbons from NMR, Raman, and Pair Distribution Function Analysis. Chem. Mater. 27, 6848-6857 (2015).

34. Harris, P. J. F. Fullerene-like models for microporous carbon. J. Mater. Sci. 48, 565-577 (2013).

35. Alam, T. M. \& Osborn Popp, T. M. In-pore exchange and diffusion of carbonate solvent mixtures in nanoporous carbon. Chem. Phys. Lett. 658, 51-57 (2016).

36. Stallmach, F. et al. NMR studies on the diffusion of hydrocarbons on the metal-organic framework material MOF-5. Angew. Chem. Int. Ed. Engl. 45, 2123-6 (2006).

37. Noda, A., Hayamizu, K. \& Watanabe, M. Pulsed-Gradient Spin - Echo 1H and 19F NMR Ionic Diffusion Coefficient, Viscosity, and Ionic Conductivity of Non-Chloroaluminate Room-Temperature Ionic Liquids. $J$. Phys. Chem. B 105, 4603-4610 (2001).

38. Every, H. A., Bishop, A. G., Macfarlane, D. R., Ora, G. \& Forsyth, M. Transport properties in a family of dialkylimidazolium ionic liquids. Phys. Chem. Chem. Phys. 6, 1758-1765 (2004).

39. Hou, J., Zhang, Z. \& Madsen, L. A. Cation / Anion Associations in Ionic Liquids Modulated by Hydration and Ionic Medium. J. Phys. Chem. B 115, 4576-4582 (2011).

40. Kondrat, S.\& Kornyshev, A. Charging Dynamics and Optimization of Nanoporous Supercapacitors. J. Phys . Chem. C 117, 12399-12406 (2013).

41. Korenblit, Y. et al. High-Rate Electrochemical Capacitors Based on Ordered Mesoporous Silicon CarbideDerived Carbon. ACS Nano 4, 1337-1344 (2010).

42. Péan, C. et al. On the Dynamics of Charging in Nanoporous Carbon-Based Supercapacitors. ACS Nano 8, 1576-1583 (2014).

43. Redondo, E., Carretero-González, J., Goikolea, E., Ségalini, J. \& Mysyk, R. Effect of pore texture on performance of activated carbon supercapacitor electrodes derived from olive pits. Electrochim. Acta 160, 178184 (2015).

44. Itoi, H., Nishihara, H., Kogure, T. \& Kyotani, T. Three-Dimensionally Arrayed and Mutually Connected 1.2$\mathrm{nm}$ Nanopores for High-Performance Electric Double Layer Capacitor. J. Am. Chem. Soc. 20, 1165-1167 (2011).

45. Krachkovskiy, S. a et al. Visualization of Steady-State Ionic Concentration Profiles Formed in Electrolytes during Li-Ion Battery Operation and Determination of Mass-Transport Properties by in Situ Magnetic Resonance Imaging. J. Am. Chem. Soc. 138, 7992-9 (2016).

46. Hayamizu, K. et al. Studies on the translational and rotational motions of ionic liquids composed of N-methyl$\mathrm{N}$-propyl-pyrrolidinium (P13) cation and bis(trifluoromethanesulfonyl)amide and bis(fluorosulfonyl)amide anions and their binary systems including lithium salts. J. Chem. Phys. 133, 194505 (2010).

47. Zhang, Z. \& Madsen, L. A. Observation of separate cation and anion electrophoretic mobilities in pure ionic liquids. J. Chem. Phys. 140, 084204 (2014).

48. Gouverneur, M., Kopp, J., Wüllen, L. Van \& Schönhoff, M. Direct determination of ionic transference numbers in ionic liquids by electrophoretic NMR. Phys. Chem. Chem. Phys. 17, 30680-30686 (2015).

49. Neimark, A. V., Lin, Y., Ravikovitch, P. I. \& Thommes, M. Quenched solid density functional theory and pore size analysis of micro-mesoporous carbons. Carbon 47, 1617-1628 (2009).

50. Cotts, R. M., Hoch, M. J. R., Sun, T.\& Markert, J. T. Pulsed Field Gradient Stimulated Echo Methods for Improved NMR Diffusion Measurements in Heterogeneous Systems. J. Magn. Reson. 266, 252-266 (1989).

\section{Acknowledgements}

The authors acknowledge the EPSRC (through the Supergen consortium for A.C.F., J.M.G., and J. C.-G.), the School of the Physical Sciences of the University of Cambridge (via an Oppenheimer Research Fellowship, C.M.), the EU Graphene Flagship (A.-R.O.R.), and the EU ERC (through an 
Advanced Fellowship to C.P.G.) for financial support. This work was also supported as part of the NorthEast Center for Chemical Energy Storage (NECCES), an Energy Frontier Research Center funded by the U.S. Department of Energy (DOE), Office of Science, Basic Energy Sciences, under Award \#DE-SC0012583 (N.M.T.). We thank Paul Bayley for assistance with PFG NMR experiments and the engineering of in situ apparatus, and Andrew Sederman for assistance with initial PFG NMR experiments.

\section{Contributions}

A.C.F prepared supercapacitor cells, performed PFG NMR experiments and analysed the data. J.M.G. recorded and analysed NMR experiments to determine total in-pore ion populations. N.M.T. performed initial PFG NMR experiments. A.-R.O.R. recorded SEM images and gas sorption isotherms. All authors contributed to design of the research, the discussion of the data and the writing of the paper.

\section{Additional Information}

Supplementary information is available in the online version of the paper. Reprints and permissions information is available online at www.nature.com/reprints. Correspondence and requests for materials should be addressed to C.P.G.

\section{Competing financial interests}

The authors declare no competing financial interests. 